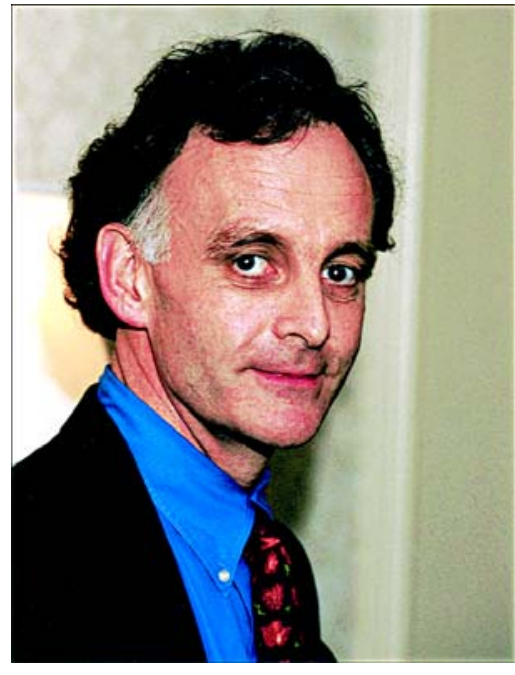

Adam Darkins, MD, MPHM, FRCS

\title{
Changing the location of care: Management of patients with chronic conditions in Veterans Health Administration using care coordination/home telehealth
}

The way that societal changes influence how health services are delivered to people with chronic diseases and conditions exemplifies how policy responses must now acknowledge patient and caregiver preferences for care. New healthcare-associated information technologies are allowing patients to choose their location for care and realize their preferences [1]. Previous long-term care policy projections had anticipated that today's healthcare system would be challenged to meet the growing need for long-term institutional care and to care for an aging population whose chronic disease incidence and prevalence would rise dramatically [2-3]. During the intervening 25 years, many factors [4-6], including reduced disability levels in the elderly, increased disposable income, changes in chronic disease patterns, and initiatives to increase caregiver and community support, have moved the long-term care equation toward noninstitutional settings. When appropriate and given the choice, many people prefer to remain living independently in their homes and/or communities and to avoid or delay placement in long-term institutional care facilities [7].

Healthcare systems must respond to a complex and continually evolving long-term care agenda that expects these systems to restructure how they provide care and, in doing so, develop noninstitutional care services that reach directly into patients' homes. Among the noninstitutional care services routinely delivered by the Veterans Health Administration (VHA) is care coordination. Care coordination has been explicitly designed to meet the changing healthcare needs of a veteran population that is aging and coping with the limitations imposed on their lives and longevity by chronic diseases and conditions, e.g., diabetes, chronic heart failure (CHF), spinal cord injury (SCI), posttraumatic stress disorder (PTSD), depression, chronic obstructive pulmonary disease (COPD), stroke, multiple sclerosis (MS), and hypertension. VHA defines care coordination as-

the use of health informatics, telehealth, and disease management to enhance and extend care and case management activities to facilitate access to care and improve the health of designated individuals and populations with the specific intent of providing the right care in the right place at the right time (http://www.va.gov/occ/).

VHA uses a range of telehealth technologies to support three classes of care coordination programs in its 154 Department of Veterans Affairs medical centers. Care coordination/home telehealth (CCHT) programs use telehealth technologies, including digital cameras, videophones, messaging/ monitoring devices, and telemonitors, to coordinate care directly from a 
patient's home. Care coordination/general telehealth (CCGT) programs use videoconferencing technologies to provide clinical services, e.g., telemental health between hospitals and community-based outpatient clinics (CBOCs). Care coordination/ store-and-forwards (CCSF) allow VHA to provide teleretinal imaging, dermatology, wound care, and pathology services to rural and remote locations. CCHT, CCGT, and CCSF enable VHA to make specialist care, e.g., rehabilitation, more widely available and accessible to veteran patients, especially those who find access to care challenging because they live in remote or rural areas. Care coordination enhances care by changing its location to a more accessible site or supporting the ongoing provision of care at a current site in which, for example, recruitment of specialist staff such as eye care professionals is problematic, e.g., in a remote or rural location.

Fundamental to VHA's ability to change the location of care by reconfiguring face-to-face care and introducing services that extend into patient's homes, community settings, and CBOCs has been the full implementation of a comprehensive computerized health record (CHR). Currently, the patients with chronic diseases to whom changing location of care applies most are those at risk of long-term institutional care placement. In healthcare organizations without a CHR (and in the VHA prior to the introduction of CHR) the charts/medical records for these patients are typically maintained in several volumes that are not reliably available because they are concurrently being processed elsewhere in the system. Even during an unscheduled clinic visit by a patient whose paper chart is available, a provider who is not familiar with the patient cannot always easily and quickly access the information in a busy clinic. In VHA's experience, an electronic record with structured, necessary health information (e.g., complaints, progress notes, medication, allergies, past history, laboratory results, and radiology) is a vital prerequisite to viable, safe, and effective remote decision making in the home and clinic. Hereafter, this guest editorial will focus on the role that CCHT plays in coordinating care.
The Institute of Medicine (IOM) considers care coordination and implementation of new information technologies one possible answer to the challenge of the rising prevalence of people with chronic diseases [8]. In espousing these IOM solutions within its care coordination programs, VHA has sought to make the home the preferred care location. VHA care-coordination programs are explicitly patient-centric and predicated on the Wagner model of chronic care [9] that encourages patient self-management [10]. Increasingly, patients want to be involved in care management decisions [11] but in routine services, this is a complex undertaking [12], especially for patients who need longterm care and have disabilities [13-14]. The finding that elderly patients with cognitive deficits can express their preferences [15] further supports the movement of care delivery away from older paternalistic approaches and encourages the development of long-term care services that incorporate new models of independent living [16]. This change of emphasis affects funding arrangements for care [17]. Therefore, because both clinical and financial considerations shape the long-term care sector, VHA considered both issues when it created carecoordination services that are patient-focused and positively address the fragmented care that adversely affects patient safety [18-19].

VHA designed its CCHT programs for noninstitutional long-term care services using a systems approach that incorporates patient preference, assesses patient's healthcare needs, and ensures safe and efficient provision of care for patients with chronic diseases. VHA has developed a tool kit that standardizes its CCHT approach. CCHT offers an information technology infrastructure and organizational framework for providing patient-focused services that help manage the biopsychosocial problems associated with care delivery in rural areas [20-21]. No evidence supports the value of routine outpatient clinic appointments in the management of chronic disease. On the contrary, patients frequently deteriorate before or after these routine clinic appointments. VHA initial pilots of CCHT were implemented based on the hypothesis that programs of care that continually monitored patients 
with chronic disease would reduce hospital admissions, bed days of care, and emergency room attendances. Outcomes analyses of CCHT in VHA have shown reduced hospitalizations, 30 percent fewer emergency room visits, a 30 percent reduction in the average number of bed days of care, improved health-related quality of life [22], and benefits in terms of satisfaction [23], use clinical outcomes [24-25], and cost. CCHT supports rehabilitation in the home and improved instrumental activities of daily living and cognitive function [25].

VHA now has CCHT programs in all 21 regional administrative units (Veterans Integrated Services Networks). As of April 2006, VHA CCHT programs support the care of more than 12,000 veteran patients with chronic conditions in their homes-patients who would otherwise need long-term institutional care. VHA's current trajectory for CCHT expansion was based upon an April 2002 needs assessment that showed how noninstitutional care delivery to this population could be accomplished with home telehealth technologies [26]. Short-, medium- and longterm implementations of CCHT were recommended. In October 2006, VHA will complete its short-term CCHT implementation and thereby will provide care coordination to 21,000 veterans with CHF, COPD, diabetes, and PTSD. These are patients for whom annual costs of care exceed $\$ 25,000$.

The medium-term implementation strategy will target 50,000 patients by enrolling patients with SCI, depression, mild dementia, MS, and Parkinson's disease. Conditional on the outcomes of the short- and medium-term implementations, VHA will consider a long-term implementation that will support the 41 percent of the chronic-disease population with low or limited activities of daily living. These 1.3 million patients, if not managed early in their disease state, will become the high-cost, highrisk, high-use chronic care patients of the future. CCHT may delay this deterioration and enable patients who want to live independently in their homes to continue to do so. VHA's strategy for supporting the care of this group is the patient-held record MyHealtheVet (http://www.myhealth.va.gov/); the patient education resources that MyHealtheVet provides will support patient self-management.
VHA systematically captures CCHT clinical activity for workload, cost, and outcomes research by integrating the health information derived from the home and feeding it directly into the CHR. In essence, VHA is developing a continuous longitudinal patient record to capture disease-related data. The current non-CCHT routine collection of clinical data is episodic and provides information for when a patient is seen in the outpatient clinic or admitted to the hospital. The CCHT data from the home are risk-stratified for the care coordinators in terms of the patients' symptoms, knowledge, and behaviors in relation to their disease/condition. VHA CCHT programs are therefore designed not only to promote patient self-management but also to act as fertile sources of outcomes research on factors influencing the clinical course of patients with chronic diseases and the optimization of their care.

VHA has established a national CCHT training center in Lake City, Florida. Through a combination of face-to-face and virtual training modalities, more than 2,000 VHA clinical staff have received CCHT training from a set curriculum. VHA, therefore, has staff with the skills and competencies to support current and planned program expansions.

As an integrated healthcare system with an aging patient population to treat, VHA has a pressing patient care delivery need that provides a clear rationale for reconfiguring its healthcare services to support patients with chronic disease and enable them to live independently in their homes. The future projections for long-term care in the United States are uncertain [27]. VHA has found that home telehealth that supports patients who are living independently in their homes or local communities is a flexible and cost-effective way of providing noninstitutional care to selected populations of patients with chronic conditions. Ensuring that these services are appropriate, safe, effective, and cost-effective requires more than just technology [28-29]. To meet the complex care needs of this population in a robust and sustainable manner, VHA has undertaken this health services development program within a care-coordination model that extends and enhances care and case management through information technologies. A guiding 
principle is that the care drives the technology, not vice versa, and thereby the services that VHA is developing center explicitly on the patient and respond to patient preferences.

\section{Adam Darkins, MD, MPHM, FRCS}

Chief Consultant for Care Coordination, Department of Veterans Affairs, Washington, DC

Email: adam.darkins@va.gov

\section{REFERENCES}

1. Kassirer JP. Patients, physicians, and the Internet. Health Aff (Millwood). 2000;19(6):115-23. [PMID: 11192394]

2. Gruenberg EM. The failures of success. Milbank Mem Fund Q Health Soc. 1977;55(1):3-24. [PMID: 141009]

3. Verbrugge LM. Longer life but worsening health? Trends in health and mortality of middle-aged and older persons. Milbank Mem Fund Q Health Soc. 1984;62(3):475-519. [PMID: 6566016]

4. Waidmann TA, Liu K. Disability trends among elderly persons and implications for the future. J Gerontol B Psychol Sci Soc Sci. 2000;55(5):S229-37. [PMID: 10985301]

5. Cutler DM. Declining disability among the elderly. Health Aff (Millwood). 2001;20(6):11-27. [PMID: 11816649]

6. Allaire SH, LaValley MP, Evans SR, O’Connor GT, KellyHayes M, Meenan RF, Levy D, Felson DT. Evidence for decline in disability and improved health among persons aged 55 to 70 years: The Framingham Heart Study. Am J Pub Health. 1999;89(11):1678-83. [PMID: 10553388]

7. Walker DM. Long-term care: Aging Baby Boom generation will increase demand and burden on federal and state budgets. Washington (DC): U.S. General Accounting Office; 2002.

8. Institute of Medicine. Crossing the quality chasm: A new health system for the 21st century. Washington (DC): National Academic Press; 2001.

9. Wagner EH, Glasgow RE, Davis C, Bonomi AE, Provost L, McCulloch D, Carver P, Sixta C. Quality improvement in chronic illness care: A collaborative approach. Jt Comm J Qual Improv. 2001;27(2):63-80. [PMID: 11221012]

10. Warsi A, Wang PS, LaValley MP, Avorn J, Solomon DH. Self-management education programs in chronic disease: A systematic review and methodological critique of the literature. Arch Intern Med. 2004;164(15):1641-49. [PMID: 15302634]

11. Fowler FJ Jr, Barry MJ, Lu-Yao G, Wasson J, Roman A, Wennberg J. Effect of radical prostatectomy for prostate cancer on patient quality of life: Results from a Medicare survey. Urology. 1995;45(6):1007-13; discussion 1013-15. [PMID: 7771002$]$

12. Kane RA, Degenholtz HB, Kane RL. Adding values: An experiment in systematic attention to values and preferences of community long-term care clients. J Gerontol B Psychol Sci Soc Sci. 1999;54(2):S109-19.

[PMID: 10097781]

13. Institute of Medicine. Improving the quality of long-term care. Washington (DC): National Academic Press; 2001.

14. Noelker LS, Harel Z. Linking quality of long term care and quality of life. New York (NY): Springer; 2001.

15. Feinberg LF, Whitlatch CJ. Are persons with cognitive impairment able to state consistent choices? Gerontologist. 2001;41(3):374-82. [PMID: 11405435]

16. DeJong G, Batavia AI, McKnew LB. The independent living model of personal assistance in national long-term care policy. Generations. 1992;89-95.

17. Feder J, Komisar HL, Niefeld M. Long-term care in the United States: An overview. Health Aff (Millwood). 2000; 19(3):40-56. [PMID: 10812780]

18. Leape LL. Error in medicine. JAMA. 1994;272(23): 1851-57. [PMID: 7503827]

19. Denison A, Pierce JR Jr. Systems analysis of a clinical error. J Public Health Manag Pract. 2003;9(1):43-46. [PMID: 12552929]

20. Demiris G, Shigaki CL, Schopp LH. An evaluation framework for a rural home-based telerehabilitation network. J Med Syst. 2005;29(6):595-603. [PMID: 16235812]

21. Prince TR, Croghan JE, Sheridan PH Jr, Weatherly JD. Enhancing efficiency and quality of ambulatory care through telehealth technology. J Ambul Care Manage. 2005;28(3): 222-29. [PMID: 15968214]

22. Chumbler NR, Neugaard B, Kobb R, Ryan P, Qin H, Joo Y. Evaluation of a care coordination/home-telehealth program for veterans with diabetes: Health services utilization and health-related quality of life. Eval Health Prof. 2005;28(4):464-78. [PMID: 16272426]

23. Wakefield BJ, Buresh KA, Flanagan JR, Kienzle MG. Interactive video specialty consultations in long-term care. J Am Geriatr Soc. 2004;52(5):789-93. [PMID: 15086663]

24. Huddleston M, Kobb R. Emerging technology for at-risk chronically ill veterans. J Healthc Qual. 2004;26(6): 12-15,24. [PMID: 15603089]

25. Chumbler NR, Mann WC, Wu S, Schmid A, Kobb R. The association of home-telehealth use and care coordination with improvement of functional and cognitive functioning in frail elderly men. Telemed J E Health. 2004;10(2):129-37. [PMID: 15319042]

26. Darkins A. The development and expansion of home telehealth in VHA. Washington (DC): Department of Veterans Affairs; 2002.

27. Singer BH, Manton KG. The effects of health changes on projections of health service needs for the elderly population 
of the United States. Proc Natl Acad Sci USA. 1998; 95(26):15618-22. [PMID: 9861019]

28. The sixth report of the Joint National Committee on prevention, detection, evaluation, and treatment of high blood pressure. Arch Intern Med. 1997:157(21):2413-46. [PMID: 9385294] Erratum in: Arch Intern Med. 1998:158(6)573.
29. Clark CM, Fradkin JE, Hiss RG, Lorenze RA, Vinicor F, Warren-Boulton E. Promoting early diagnosis and treatment of type 2 diabetes: The National Diabetes Education Program. JAMA. 2000:284(3):363-65. [PMID: 10891969$]$

DOI: 10.1682/JRRD.2006.03.0029 